\title{
Using Research to Inform Health Policy: Barriers and Strategies in Developing Countries.
}

\section{OPPORTUNITIES AND CHOICES WORKING PAPER NO. 9 MARCH 2004}

Monique Hennink, Ph.D.*

Opportunities and Choices Reproductive Health Research Program

Division of Social Statistics

School of Social Sciences

University of Southampton

Southampton S017 1BJ

United Kingdom

Tel: +44-2380-597989

Fax: +44-2380-593846

Email: mon@socsci.soton.ac.uk

Rob Stephenson, Ph.D.

Bill and Melinda Gates Institute for Population and Reproductive Health

Department of Population and Family Health Sciences

Johns Hopkins Bloomberg School of Public Health

615 N Wolfe Street, W4508, Baltimore, Maryland

21205-2179 USA

Tel: +1-410-9552103

Fax: +1-410-9550792

Email: rstephen@jhsph.edu 


\begin{abstract}
This paper examines the dissemination and uptake of health research into policy and program delivery in four developing countries. In-depth interviews were conducted with health researchers, policymakers and practitioners at both local and national level.
\end{abstract}

The study highlights the similarities across the study countries in the barriers to effective dissemination and uptake of research results. A fundamental barrier to the uptake of research by decision-makers is the lack of appreciation of the important contribution that research can make to policy and program development. A further barrier is researcher's lack of appropriate 'packaging' of research findings which consider the needs of different policy audiences. Dissemination within academic circles also restricts access by decision-makers and practitioners. Overcoming the barriers requires effort on behalf of researchers, decision-makers and donor agencies. The strong presence of donor agencies in developing countries places them in a position to both enable and to encourage dissemination activities and communication between researchers and policymakers or practitioners. Increased collaboration between all three parties is one of the key strategies towards increasing the uptake of research into health policy and program development. 


\section{Introduction}

Research is generally understood to be a systematic process for generating new knowledge, and can act as a powerful tool for providing information for policy formation (Walt 1994). The continuing trend towards evidence-based policy formation has increased the demand for research outputs that can provide clear, concise policy-relevant findings (WHO 2000; ECDPM 2000). A prerequisite for evidence-based policy formation is the timely provision of scientifically sound and up-to-date information to policymakers (WHO 2000; Bark 1979), and the success of a country's development efforts depend upon the degree to which its planners and program managers use and apply research for decision-making (Torres 1981). Demographic research continues to produce a large body of scientific findings that can address critical issues faced by policymakers, and informed policymaking can benefit from understanding the policy implications of such research (RAND 1997). The extent to which such research is translated into policy action, however, is dependent on the success of communicating research outputs between researchers and policymakers.

The effective dissemination of research results to policymakers is an essential element of any research program, not only as a means of translating research results into policy action, but also to provide 'pay-back' for the investment in health research (Askew 2002). The growth of large donor-funded operations research programs focused towards developing countries has increased the need to provide evidence of policy impact, which in turn has placed greater importance on ensuring that research outputs are communicated effectively to policymakers. Effective communication between researchers and policymakers, however, provides a continual challenge. 
Previous research has identified the existence of a gap between the amount of research that is produced and the amount that is implemented in policy development and program delivery, resulting in the current under-utilization of health research (Walt 1994b; RAND 1997).

This paper examines issues of communication across the researcher-policymaker interface in four countries: Malawi, Tanzania, India and Pakistan. Although previous research has identified the existence of a communication gap between researchers and decision-makers (Walt 1994;1994b), little is known of the factors creating this divide. This paper explores current modes of research dissemination between researchers and policymakers, and examines the barriers to effective communication between the two

parties. An understanding of the difficulties faced by researchers and policymakers in disseminating and utilizing research outputs has the potential to increase our knowledge of the communication process, and to highlight strategies to improve the dissemination and uptake of research outputs.

\section{Models of Research Communication}

Research results can inform and be used by a wide variety of decision-makers, such as politicians, public officials, program implementers, non-government agencies, international organizations or service delivery bodies. Policy decisions can also vary from Government ministries developing national population policies at one end of the spectrum to informing program delivery strategies at local service points at another level. Due to the variation in scope and level of decision-makers, research results need to be communicated differently to each policy audience and according to the type of policy, decision, or program being influenced. 
Previous studies of research utilization have used a similarly broad definition of policymakers, which has contributed to the development of a conceptual framework to describe the use of research in health policy formation (Hanney et al 2002; Hanney et al 2000; Buxton and Hanney 1996). The framework consists of two elements: a categorization of the potential benefits of using research in health policy formation, and a description of the stages involved in the utilization of research in policymaking. The stages include the inputs to research, the research process, primary outputs from research, secondary outputs from research, practitioners application of research and final outcomes. Although the stages are presented as a linear process the framework also includes various feedback loops and forward leaps, recognizing that the process of research utilization is often multidirectional (Hanney et al 2002). The following models of research utilization more fully describe the various processes of interaction between researchers and policymakers.

Models of research utilization in policy development fall into three broad categories; rational models, incremental models and political models ${ }^{1}$ (Hanney et al 2002: Weiss 1979; 1980). Rational models of research utilization include the knowledge driven model, which describes research as a linear sequence whereby research generates knowledge that implies action and research is passed to policymakers as a final stage in the research process (Walt 1994; Weiss 1977). The problem-solving model also follows a linear sequence, but the research issue is identified by the end-user in a deliberate attempt to answer a policy question (Hanney et al 2002). Porter and PrysorJones (1997) argue that such deliberate dissemination of research is most likely to

\footnotetext{
${ }^{1}$ The categorization of models is taken from Hanney et al (2002). In their paper, Hanney et al (2002) state that this is not intended to be a comprehensive categorization of models of policymaking, but is based on previous analyses of public policymaking that focused specifically on the process of research utilization (Kogan and Tuijnman 1995).
} 
result in policy change as the stimulus to seek information arises directly from a specific policy problem.

Incremental models have a longer timeframe for research utilization and include the interactive model and the enlightenment model. The interactive model involves a number of interactions between researchers and policymakers throughout the research process, and enables each party to be exposed to the world of the other. Hanney et al (2002) state that increasing attention is focusing on the concept of interfaces between research producers and users, and that research is less likely to be used when there is no interaction across this interface. They go on to state that interaction needs to be considered at various stages in the research process, including priority setting, commissioning of research and communication of findings. The enlightenment model argues that research outputs permeate gradually into the policy process through a number of indirect information channels (Walt 1994), and that it is not a single piece of research but a cumulative weight of information which leads to policy change (Weiss 1977). Smith (1993) argues that this model provides a more realistic view of the researcher-policymaker interface, which acknowledges the dynamic exchange of information between a range of channels and actors.

Political models include the political model, whereby policymakers use research as ammunition to support certain political points of view and to refute others. Secondly, the tactical model of research utilization is used when there is pressure for action to be taken on specific issues that leads to the commissioning of research on the topic (Martin 2002). Hanney et al (2002) state that such commissioning of research can 
lead to more informed policy making, rather than shotgun policy development in response to the pressure to respond.

\section{Methodology}

This research was conducted in four countries: Malawi, Tanzania, Pakistan and India. Asian and African countries were selected to identify whether there exist broad continental differences in the issues under investigation, and whether country-specific issues are evident. Information was collected via in-depth interviews with both health researchers and policymakers responsible for developing health policy, as shown in Table 1. Preliminary investigations within each study country identified the range of organizations conducting health research (i.e.: university-based researchers, independent institutions), or involved in health policy development (i.e.: Government ministries, INGOs). Each organization was invited to participate in the study via a letter or personal contact. The selection of different types of research or policy organizations enabled a wide range of views and opinions from differing perspectives to contribute to the study. A total of 48 in-depth interviews were completed; which comprised of 12 interviews in each country with six researchers and six policymakers or practitioners.

Information was collected via in-depth interviews using a semi-structured discussion guide. The interviews covered the following topics; current practice of research dissemination and uptake; effectiveness of current dissemination strategies; barriers to the dissemination of research; and suggested strategies for improved dissemination. All interviews were tape-recorded and fully transcribed. Textual data analysis was conducted using the principles of Grounded Theory (Glaser and Strauss 1967; Bartlett 
and Payne 1997), whereby the textual data are categorized by themes within the data and examined across the whole data set. Common themes contribute to developing a picture of collective experience (Taylor and Bogdan 1984), and themes identified by specific subgroups (ie: researchers or policymakers) are further examined for contextual explanation of the issues. Verbatim quotations from respondents are used to illustrate key themes from the respondent's own perspective.

\section{Results}

Due to the similarity of issues across the differing contexts of the research, the results are presented by issues rather than country-specific results. The results are structured according to the barriers experienced by researchers, by policymakers and a range of common barriers.

\section{Current Methods of Dissemination and Uptake of Research Results}

The range of strategies used by researchers to disseminate research outputs to policymaking audiences is similar across all study countries. Differences in the dissemination process, however, exist between research that is commissioned (by a donor or a government agency) and non-commissioned research. Respondents identified that with commissioned research there is a direct channel of communication between the researcher and the end-user, which facilitates the dissemination of the final research outputs. Respondents also stated that the commissioning agency is typically involved in the research process and has a vested interest in the research outputs and it is therefore more likely to be utilized in policy development. Additionally, commissioned research is often disseminated via donor-funded workshops, conducted with a range of in-country stakeholders, or the donor agency 
initiates the distribution of the research outputs to a wider audience. Many policymakers also reported that the research results they most frequently use are those from commissioned research. For example,

Most research I've done has been driven by the policymaker or a donor, so it's easy to pass on the findings, as from the start there was a consultation with the data users...so the donor or policymaker is part of the team, broadly speaking (researcher, Malawi).

The breastfeeding research with UNAIDS led to the National Breastfeeding Policy change and infant-friendly hospital scheme. Both UNAIDS and the Government were involved, so communication took place from the design phase onwards, this allowed it to become implemented easily. It had started from the lap of the Government...they owned it, it was not an outsider doing the research and asking for a policy change (researcher, Pakistan)

For non-commissioned research the channels of dissemination to policymakers are less clear and more varied. Many researchers stated that dissemination of noncommissioned research is limited to academic channels (e.g. papers in peer-reviewed journals or presentations at conferences). The direct dissemination of noncommissioned research to policymakers most commonly involved either the distribution of a research report to a range of policymakers or inviting key policymakers and other stakeholders to a dissemination workshop. 
Policymakers reported seeking research findings only when they had specific information needs. If the information is not available internally or through commissioned research outputs, policymakers consulted a range of sources including other ministries and government departments, documents from international research organizations or national data sets. To a lesser extent, policymakers contacted university departments and national research organizations; however, this channel was only used if there was an established link with the organisation. In many cases consultants were employed to locate relevant published material or to conduct a research study.

Both researchers and policymakers reported that there exist no common, structured mechanisms through which to disseminate effectively non-commissioned research; this is highlighted by the disparate strategies of dissemination used by researchers and the sources of uptake of research used by policymakers. However, for commissioned research, there is a direct channel of communication between researchers and policymakers.

\section{Researcher's Barriers to Dissemination of Research Outputs}

\section{Policymaker's Perceptions of Research}

Researchers reported that the lack of a strong evidence-based culture in policy development was a significant barrier to the uptake of research by policymakers. Researchers perceived that research is given low priority by policymakers and that research findings are not valued in policy formation. As a result research is often seen to have little contribution to the policy development process, and policymakers are seen as not fully appreciating the potential contribution of research in enhancing 
policy formation. In addition, researchers felt that research is perceived as an unnecessary expenditure for policy development in resource poor countries. These issues are highlighted by researchers in the following extracts;

The ministers make the policies themselves, without using what we send them, they don't realize that research could help them (researcher, India)

Policymakers don't see the role that research plays in everyday situations. Senior government officials don't appreciate the role of research in programmes (researcher, Malawi).

The resistance is big basically because most policymakers don't think that research is essential for their policies. There is a general feeling among policymakers that as far as policymaking goes they are the experts. If you want to bring in researchers they are just there to punch in numbers (researcher, Pakistan).

Most of the policies in Pakistan have been developed without the use of research...for example, maternal mortality...abortion and violence against women (researcher, Pakistan).

Researchers also suggested that policymakers may not fully understand how to use research to support policy formation. They noted that policymakers may not have the ability to evaluate the quality of a research study or to interpret research findings, thus experiencing difficulties in incorporating research findings into policy development or 
into service delivery programs. This may lead to the failure to translate research into policy or to extraneous conclusions drawn from research results. For example;

They (policymakers) do not understand the research process, so they do not differentiate between good and bad research...many of them don't even understand the difference between qualitative and quantitative research (researcher, Pakistan)

Policymakers may not understand research. They feel that it may take years to get an outcome, they are interested in outputs for today. There might be a lack of vision on the part of the policymaker (researcher, Tanzania)

\section{Emphasis on Statistics}

While researchers recognized the importance of measurement indicators, and the increasing pressure on policymakers to justify programs or quantify changes, they felt that policymaker's focus on statistics under-utilized research results. Researchers felt that substantive issues arising from the research could also impact on policy. Policymakers themselves acknowledged their focus on statistical information from research outputs. The following comments illustrate these points;

They (policymakers) are interested in a few indicators, for example, what is the CPR? Which they have to report to their highers, but other areas that are really important such as quality and side effects are not given as much attention (researcher, Pakistan).

Basically it's statistical information we require from the researcher because they have it readily available and can provide it promptly (policymaker, Pakistan). 


\section{Lack of Dissemination Skills and Access to Policymakers}

A key theme raised by many researchers is their lack of skills in disseminating research to policy audiences. Researchers highlighted their lack of training in communication of research findings outside academic circles. They were also uncertain about whom to disseminate research findings to, and expressed difficulties in identifying and accessing policymakers. Although some researchers were able to identify policymakers, they reported that the frequent changes in government portfolios meant that developing and maintaining links with policymakers was problematic. A Malawian researcher highlights these points; Researchers are not trained to communicate with policy people. The focus is to write papers for publication for a completely different audience. Few researchers know about the difference of writing for policymakers, so they submit big reports.

\section{Lack of Resources}

Another fundamental barrier identified by researchers is the lack of resources for dissemination activities, particularly in Malawi and Tanzania, where researchers reported that often there are no funds available to consider dissemination activities beyond the distribution of a research report. Whilst resource issues were less apparent for donor-funded research, for Government sponsored or independent research, resource constraints often curbed dissemination activities. For example; How dissemination works will depend on the amount of resources, resources for dissemination are limited, so you can't disseminate in the way you would like to due to shortages, and we don't have PowerPoint packages to present findings nicely (researcher, Malawi). 


\section{Donor-Researcher Relationship}

The prominence of donor-driven research, particularly in Malawi and Tanzania, raised a number of issues related to dissemination. First, researchers highlighted the common disparity between the health issues investigated by international donor agencies and the priority areas of national policymakers, hence policymakers often disregard research outputs that are not focused on national areas of priority, for example;

These researchers get funding which has been specified to be targeted at specific areas, but that is not what the policymakers really need (policymaker, Tanzania).

Much research is conducted by external consultants and policymakers are not involved...so are not aware of the issues and sometimes don't have any interest in the research (researcher, Malawi).

Second, researchers are typically involved in donor-initiated research as consultants and are not obliged to become involved in dissemination activities. Thus, a protocol has developed whereby researchers pass research findings only to the donor agency and then move on to the next consultancy. For example; We are not authorized to give out that report, only to give it to the sponsor - either the Government of Pakistan or the donor agency (researcher, Pakistan). In this situation, the onus is on the donor agency to disseminate research outputs more widely. It was felt that involving researchers in the dissemination process would be beneficial as they were more aware of the context of the issues brought out in the research. 
Third, research conducted by international agencies that are based outside the study countries often has limited time in-country, which is often tied to strict fieldwork timelines. There is often little or no time for the researchers to consult national policymakers to refine research proposals that embrace local research needs or to discuss the policy or program implications of the research findings with ministries or health providers. Researchers felt that the drawback of this situation was an inability for research organizations to; identify the research priorities within each country; understand the cultural and contextual issues surrounding the research; and develop policy recommendations that reflect 'on the ground' realities. Such research outputs were also most likely to be presented at international conferences or published in international journals, and hence are not easily accessible to policymakers and government ministries in the study countries. The following comments reflect these issues;

Research is donor-driven rather than created through need. The policymakers don't take these research recommendations seriously...external consultants also are not aware of Malawi culture and so annoy those who are responding to the research (researcher, Malawi).

International agencies give a background but local agencies give the context of India. International agencies should have liaison with Indian organizations to understand the complexities on the ground (policymaker, India). 


\section{Policymaker's Barriers to Uptake of Research Outputs}

\section{Limited Access to Research Outputs}

Accessing research outputs is one of the greatest difficulties faced by policymakers in utilizing research findings. Policymakers reported that research results often do not reach ministries, government officials or health practitioners as they are disseminated in academic circles or passed only to the donor agency. Policymakers stated that they seldom access these sources. Policymakers requested increased in-country dissemination of research outputs to wider target audiences. The following comments support this issue;

The research that has been conducted is usually by the academics or the universities, and is published in the international journals and so they don't get shared at the local level or the country level (policymaker, Pakistan).

Researchers do research for dissemination in publications or technical papers where policymakers don't get the chance to read. There is still a gap in utilizing the available research into programming or policymaking (policymaker, Tanzania).

\section{Lack of Central Source of Research Outputs}

An issue identified mostly by policymakers in Malawi and Tanzania is the lack of a central depository for health research outputs. For example, There is no inventory of research available. Sometimes you find out about Tanzanian research while at a conference abroad. There is more available abroad than in Tanzania. There's no systematic way of finding out about research, or if available it's not publicized 
(policymaker, Tanzania). Policymakers identified that a common consequence of the absence of a central source of research outputs is the duplication of research on the same issues. A depository of research outputs was thought to be particularly useful for accessing unpublished studies. It was suggested that such a depository be co-ordinated by a public institution to enable greatest access and should be publicized widely amongst policymakers and health practitioners

\section{Quality of Research}

The quality of in-country research was an issue that discouraged some policymakers from using local research outputs and prompted them to seek research findings from international agencies. This was a particular issue in Malawi and Tanzania, where policymakers identified that the small skill base amongst local researchers made it difficult to conduct high quality research. Policymakers felt that the lack of government investment in the research sector meant that local researchers have not had the advantage of expert training afforded to those in international agencies, and so the technical competence to undertake research of an international standard was not fully developed. For example;

There are quality issues with in-country research; greater validity is given to research conducted by international agencies, as long as they used local people to field it (policymaker, Malawi).

The difference in quality of local based research versus internationally conducted research is important. Malawi based research does not have the advantage of technology to present results in a digestible form. Even substantive research is more 
traditional scientific enquiry rather than research for programmers' needs. International NGOs have worked hard at developing ways to approach policymakers (policymaker, Malawi).

\section{Mutual Barriers to Communication}

\section{Lack of Formal Communication Channels}

Both researchers and policymakers identified the lack of formal channels of communication as a barrier to effective dissemination and uptake of research results. Policymakers expressed difficulties in identifying researchers beyond informal contacts, and researchers faced problems in identifying policymakers to whom to disseminate research outputs. Both groups felt that a communication forum (such as a research association or meeting forums) would enable effective dialogue between researchers and policymakers. To foster more effective communication between researchers and policymakers, it was suggested that researchers be included on Ministerial advisory boards to identify priority research areas and define appropriate research agendas to allow research activities to respond to programmatic needs. Large research organizations in Pakistan kept a list of key contacts in various Ministries and Government institutions to facilitate communication and dissemination of research. Similarly, in Tanzania it was suggested that the university hold a consultancy list of researchers and topics of specialization to facilitate easier identification by policymakers. 


\section{Lack of Collaborative Research}

Policymakers reported that when they were involved in the research process, had commissioned the research, or the research was in direct response to a policy need, it was more likely to be utilized for policy development. Researchers also reported that the involvement of policymakers in the research process led to a more effective consideration of policy issues, political limitations and practical realities in implementing the research findings. Both parties, however, reported that much research was conducted without collaboration and this posed a significant barrier to dissemination and utilization of research results. Collaboration between researchers and policymakers was encouraged at various stages in the research process; in defining research proposals, designing research questions, and particularly in shaping policy recommendations that are realistic and relevant to the resource constraints of the ministries. Ensuring that policymakers gain a sense of ownership of the research is seen as crucial to the uptake of findings, illustrating the importance of developing a relationship of trust between researchers and the policy community. The following extracts are typical of many comments that illustrate the importance of collaboration. There needs to be a whole dialogue between policymakers and researchers at the beginning of the research study, so that it becomes something that programmers have a vested interest in and researchers understand that vested interest and try to meet it. That might help to facilitate the uptake of research findings in decision-making (policymaker, Malawi)

As a programmer, if I am involved in the research I will be able to understand it better, similarly if a researcher can participate in the policymaking level then he will be able to make an impact in a better way (policymaker, India) 
...the Reproductive and Child Health Study, we went back to the policymakers as a research team, shared the results and got ideas on how to introduce the strategy and came up with a plan jointly. Going back to the policymakers is important, to enter a dialogue... to involve the policymakers (researcher, Tanzania)

It's easier to pass on results when the policymaker initiates the research. They are more likely to listen because they have ownership. Effective communication between researchers and policy people is how the ownership of a particular piece of research is established (researcher, India)

\section{Format and Interpretation of Research Findings}

Many policymakers and practitioners reported difficulties with the format and style in which research outputs were presented, stating that research reports were often written in an academic style using technical language, and include complex statistics that are difficult to understand. Policymakers stated that research outputs were often too lengthy and that concise, well-structured reports with an executive summary of the key findings and policy implications were more appropriate. Researchers, however, felt that summarizing research findings risked loosing the detail needed to fully understand the research problem. For example; But if the report is 100 pages, how can you make that executive summary in two pages? (researcher, Pakistan). Researchers also felt that their academic peers would poorly regard research that did not provide adequate detail of the research methodology, statistical techniques and use the correct terminology. 
One of the critical issues for policymakers in using research outputs is the frequent absence of policy implications or recommended interventions from the research. Furthermore, policy implications that are presented are often too general or unrealistic in terms of resources. Some policymakers felt that a range of policy recommendations should be provided such as short, medium and long-term strategies and that options should be given for various resource scenarios. They also felt that research reports should highlight which agencies should be responsible for initiating changes. In response, researchers felt that they are often not aware of policymakers' priorities and resource constraints and therefore find it difficult to develop feasible policy recommendations. Researchers also stated that policymakers often judge the policy recommendations simply on practicality and affordability of implementation rather than on the importance of the issue. For example;

Reports are in an indigestible form without adequate analysis of policy or programmatic implications; therefore people note the findings but don't act on them (policymaker, Malawi).

It is the how part, how you can change things, what you should do. Researchers usually don't do that, they put the research on the table and say now you figure out what to do (policymaker, Pakistan).

They (researchers) need to interpret figures and findings, not just present the finding that one third of children born to teenage mothers die - say that this means that there should be pregnancy prevention available (policymaker, Malawi) 
Sometimes researchers don't know who to address their recommendations to - they say policymakers - but that is a broad term (policymaker, India).

\section{Political Influences}

Researchers and policymakers acknowledged that policy formation is often influenced by political priorities and constrained by the resources of government. It was recognized that even well developed research findings may not be acted upon if the political climate was not conducive to change. Some researchers were reluctant to disseminate research findings that have political implications and felt that it would be inappropriate to disseminate findings that were in conflict with current national politics. While these issues did not discourage researchers from disseminating research outputs to policy and program audiences, researchers were aware that complex political issues often influenced policy development. The extracts below highlight the political barriers to dissemination.

Everyone who read the report thought it was excellent, the Ministers were very happy with it, but no one could implement it as it was too politically contentious (researcher, Pakistan)

Research findings may be beyond the capabilities of policymakers, even though the problem is highlighted by research. Our research found that many women were delivering at home due to lack of trained staff at hospitals. But policymakers are not permitted to employ more staff (researcher, Tanzania) 
Some researchers may recommend one way which may be the most effective but not the cheapest - so we cannot implement it. In developing countries need cheap and effective recommendations (policymaker, Tanzania).

\section{Discussion}

Although this study was conducted in four very different countries it is worthy to note the uniformity of issues raised by researchers and policymakers across the differing contexts, indicating the broadly common experiences in the process of research dissemination. A number of issues, however, were more prominent in the African study countries, in particular the influence of donors on the process of dissemination. Also the resource and infrastructure limitations and the smaller pool of skilled professionals in health research in Malawi and Tanzania lead to the greater prominence of issues such as the lack of communication networks, the need for central depositories of research information and the lack of in-country expertise for policy research in these countries.

A further issue in the dissemination and uptake of research is the question of who should take responsibility for the dissemination of research results. The dissemination of research and its contribution to the policy development process is clearly influenced by a range of activities and a range of players. This research has shown that the communication of research results can be improved through changes in the activities of three key groups; researchers, policymakers and donor agencies. The following discussion will highlight a range of strategies which can be undertaken by each group to foster more effective dissemination and utilization or research results in developing country contexts. 


\section{Strategies for Researchers}

The two key strategies for researchers are to give careful consideration to appropriate 'packaging' of research findings which consider the needs of different policy audiences and to widen the target audiences for research dissemination. These two points are critical when disseminating non-commissioned research, where there has been no involvement of an end-user in the research process. Researchers need to revise the traditional academic format of research reports and produce brief research summaries with key bullet points, clear policy recommendations, simple language and reduce the methodological, statistical and theoretical content. In addition, when researchers initiate dissemination they need to consider the full range of end-users of the information and target dissemination efforts to the broadest possible range of stakeholders. Research results may be relevant not only to a particular Government ministry, but may also impact on a range of service delivery practitioners, law enforcement agents (i.e. for abortion research), community groups, educative groups, non-Government organizations, as well as academic audiences and the media.

In addition, dissemination activities consume considerable time and resources and therefore need to be explicitly included in research proposals to donor agencies. Researchers need to be proactive to include a dissemination phase in research proposals which include a dissemination plan, target audiences, dissemination activities, research 'products' (i.e. fact-sheets, executive summaries, newsletter articles), the range of communication media to be used and a budget.

Researchers' lack of skills in disseminating research outside academic circles needs to be addressed through the provision of training on; communication strategies for 
differing audiences; developing a variety of research outputs; and shaping messages for policy audiences. Such training would also foster a greater awareness of how policymakers use research information and the constraints within which most policymakers operate. Training modules could also be included in the teaching curriculum at universities, to foster an appreciation of the importance of research dissemination and utilization. Such capacity building, however, would require an investment in the research sectors of the study countries.

An alternative to training researchers in dissemination is to utilize 'communication mediators' or 'knowledge brokers' who work at the interface between research organizations and target audiences. They may be advocates, communications experts and even donors, who act as the messenger for research messages or may design knowledge-transfer plans that are executed by researchers (Lavis 2003; Askew et al 2002). Such mediators promote the main findings of research to a wide audience in an attempt to promote the policy relevance of the research and focus stakeholders' attention towards specific areas of need. Askew et al (2002) argue that the increasing importance of mediators in the dissemination process has evolved both from researcher's lack of understanding of the policy process and policymakers difficulties in understanding research outputs. The use of mediators offers the potential to increase policymaker's access to research outputs particularly in settings with poorly developed communication between researchers and policymakers.

\section{Strategies for Policymakers and Practitioners}

A fundamental barrier to the uptake of research is the absence of a strong evidencebased culture within policy and program development, and a lack of appreciation of 
the contribution of research to the policy process. Promoting an ideological change which values research-evidence in policy development is a long term process. However, it can be influenced through closer collaboration between researchers and policy and program personnel, as discussed below.

More easily surmountable strategies for policymakers and practitioners involve the wider dissemination of commissioned research. When commissioning research, policymakers need to ensure dissemination to wide range of stakeholders, such as; other government ministries, service delivery agencies, community advocacy groups. Identifying funds for dissemination workshops in the research proposal would go some way towards enabling wider dissemination.

\section{Strategies for Donors}

The barriers to dissemination highlighted by both the research and policy community also point to a range of strategies for donor agencies in the process of research utilization. While some of the shortcomings of effective dissemination have been aimed at the donor agencies (such as lack of in-country dissemination, mismatch between donor research and policymaker priorities, limited dissemination audiences), donors also have a particularly important role in the facilitation of research dissemination. Given the strong presence of donor agencies in the study countries this places them in a position to both enable and to encourage dissemination activities and communication between researchers and policymakers or practitioners.

Donor agencies have a role to play in two areas. First, donors can encourage researchers to incorporate dissemination strategies into a research proposal, so that 
funding for project-based dissemination is available and the researchers remain involved in in-country dissemination activities. Donor agencies may also give priority to research proposals that have planned collaborative activities with key stakeholders at various stages in the research process, in particular joint development of the proposal or identification of the research problem. Second, where donor-funded research does not involve in-country research collaborators, donor agencies need to allocate project time towards consultation with the end-users of the research in the study countries, preferably at the initial stages of the research process, and establish communication with the stakeholders throughout the research process. In addition, donors should widen in-country dissemination activities to include a range of local stakeholders, to ensure that information reaches key policy audiences in the country itself.

\section{Collaborative Strategies}

The remaining strategies for improving the dissemination and uptake of research results require joint action between researchers, policymakers and donors. Research can have the greatest impact on policy when effective communication exists between researchers, policymakers and the community affected by policy change (Porter and Prysor-Jones 1997). Much research communication in the study countries, however, takes place through academic channels and so does not reach policy audiences; therefore policymakers often make decisions based on inadequate information. This underscores the importance of the research and policy community working together to establish formal communication channels and central depositories for research outputs, a suggestion also supported in other research (White 1993; Worral 1972). One example of an effective clearing house of research information is the 
Commonwealth Regional Health Community Secretariat (CRHS). One of the objectives of the secretariat is to collect relevant health research information from a variety of sources, repackage it and disseminate it to its member states in East, Central and Southern Africa (CRHCS 2003). A similar initiative will be undertaken by the Ministry of Health in Malawi, who are planning to commission a data base of research projects and researchers in the field of reproductive health.

One of the key strategies for increasing research uptake is to conduct research in partnership between the research producers and research users. From this study policymakers and practitioners assert that they are more likely to acknowledge and utilize research if the research process has been a collaborative one. It has also been shown that policy relevant research is often conducted in isolation of the end users, with only token collaboration or with contact only as a final stage in the research process.

Collaborative research requires actions on the part of researchers, policymakers/practitioners and donors. Decision-makers need to communicate their research needs and policy priorities to researchers, a strategy which has been hampered by the lack of effective communication channels and policymakers resistance to value the contribution of research in the policy process. Researchers need to involve the end-users of the information throughout the research process so that interaction becomes a continual process. Effective communication between researchers and policymakers at each stage of the research fosters a sense of ownership of the research which is critical to the uptake of findings (Porter and 
Prysor-Jones 1997; Martin et al 2002; Hanney et al 2002). Donor agencies also have a role to play in facilitating collaboration, in the ways discussed earlier.

Undertaking collaborative research partnerships would go some way to overcome the existing barriers to research utilization in the study countries. First, developing research partnerships would overcome the disparity between issues investigated by researchers/donors and policymakers' actual research priority areas. Constructive discussions can help to ensure some synthesis between the needs of in-country policymakers and the efforts of research organizations. Second, collaboration would enable decision-makers to develop a greater understanding of how to utilize research to support policy development, and to translate substantive research findings into policy issues to balance the current emphasis on statistical research findings. Such understanding may also foster an appreciation amongst policymakers for evidencebased policy and program development. Third, collaboration with the end-users of the research would enable researchers to give more effective consideration to policy and service delivery issues, political limitations and practical realities when developing strategies for implementing the research findings. Researchers would also benefit from guidance on the appropriate format and style of the research findings for policy audiences. Finally, collaboration assists in bringing together the researchers and policy community to overcome the difficulties of access to either party. However, dissemination efforts should not overlook the need to ensure findings are communicated to a wide-ranging audience. Much previous research also underscores the importance of collaborative research in increasing the likelihood of research utilization into policy (Hanney et al 2002, Martin et al 2002; Aggleton 2002; Solo et 
al 1998; Knott et al 1980; Patton et al 1977); collaboration is also the focus of the interactive model of research dissemination (Weiss 1979).

Finally, it needs to be recognized that research dissemination is often superseded by overriding influences such as the political environment and political ideologies; so that even effective dissemination of research to policymakers does not necessarily ensure that it is used in policy formation. Walt (1994) states that political environments are not always conducive to the incorporation of research into policy formation. In addition, research that advocates change may disrupt long-standing power-relationships and organizational cultures that take a great deal of effort to implement, and as such may be ignored by policymakers (Haaga and Maru 1996).

\section{Conclusion}

This study has highlighted that the barriers to effective dissemination and uptake of research results by policymakers were remarkably similar across the differing contexts of the study countries, suggesting that communication between researchers and policymakers continues to be a widespread problem and that the divide between the two parties is shaped by common factors. This study is based on qualitative data and therefore cannot be seen as representative of all researchers and policymakers views in each of the study countries. However, the similarity in the issues across the four countries points to the need for further operations research to test the effectiveness of strategies to improve communication of research across the divide. From this study it appears that overcoming these barriers in developing country contexts requires efforts on behalf of researchers, decision-makers and donor agencies. Increased collaboration between all parties is one of the key strategies 
towards increasing the uptake of research into policy and program development. Hanney et al (2002:16) suggest that "it is appropriate to focus on the actions that could be taken to encourage permeability at the interfaces between policy-makers and researchers. Such actions should help ensure both that researchers are aware of policymakers' needs, and that the policy-making system is willing and able to absorb relevant research findings." 
Table 1: Types of Respondents

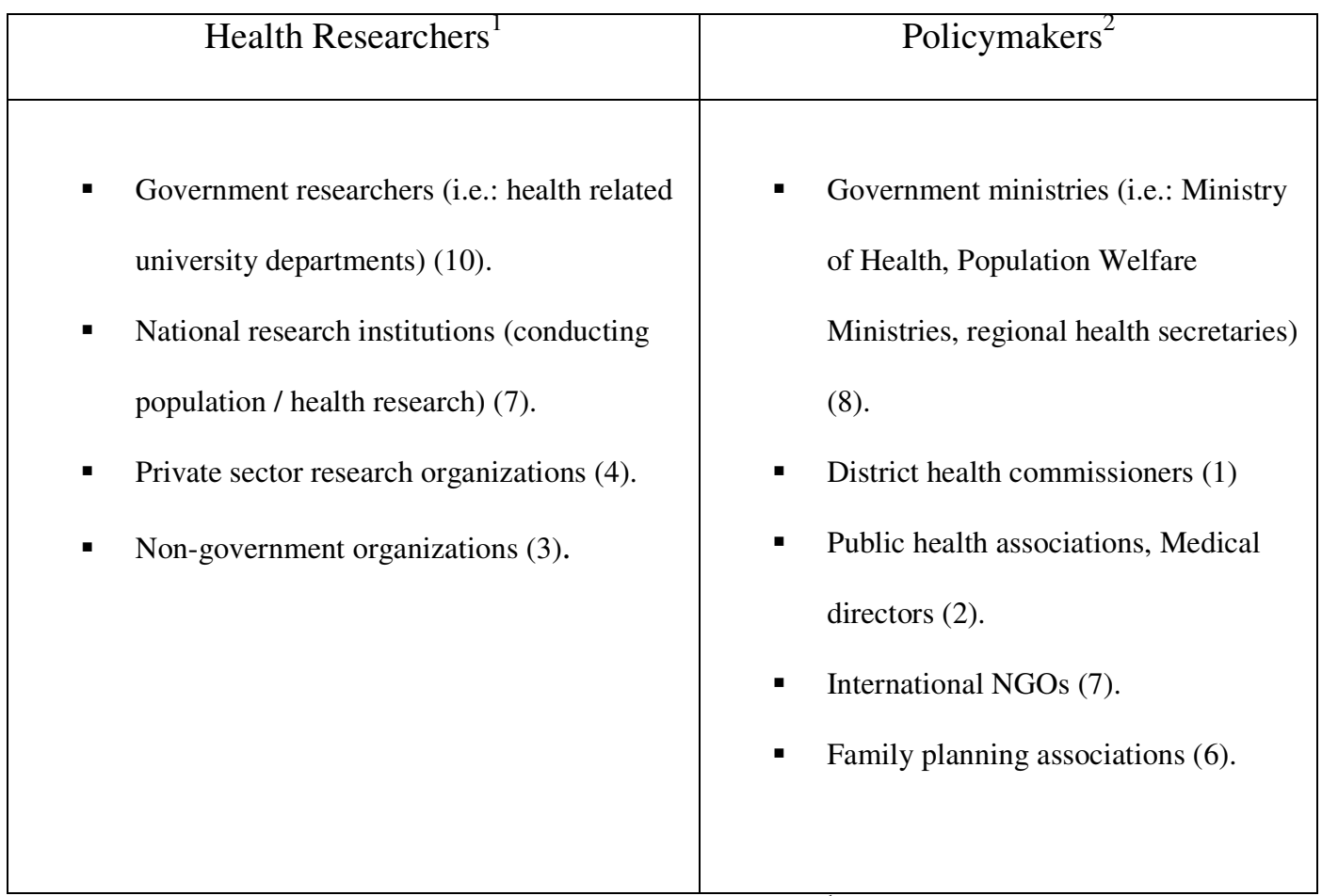

Notes: Number of respondents in each category is shown in brackets. ${ }^{1}$ Each organization was asked to identify the principle health researcher who was invited to participate in an interview. ${ }^{2}$ Key individuals directly responsible for heath policy formation at provincial or national level were interviewed. Government ministers and secretaries were identified for interview; where these were unavailable the organization identified the most relevant individual. The chief executive of each NGO health service was interviewed. 


\section{References}

Aggleton P. (2002) Promoting the Use of 'Reproductive Health Results' Towards Greater

Synergy. Workshop on Moving Beyond Research to Influence Policy, 23-24 January 2001, University of Southampton, Department for International Development, UK, Population Council/FRONTIERS, Safe Passages to Adulthood.

Askew, I, Z. Matthews and Partridge, R. (2002): Going Beyond Research: A Key Issues Paper Raising Discussion Points Related to Dissemination, Utilisation and Impact of Reproductive and Sexual Health Research. Drawn from a two-day Meeting of Researchers and Policymakers. http://www.socstats.soton.ac.uk/choices/workshop.

Bark, D.S. (1979): Overview of Research Utilisation for Population Program Development from the Policy Analysis Perspective. A Strategy for Research Utilisation on Population and Family Planning. Korean Institute for Family Planning, Seoul, Korea.

Bartlett, D, and Payne, S. (1997): Grounded Theory- Its basis, rationale and understanding. Chapter 13 p173-195 in McKenzie, G., Powell, J., and Usher, P. Understanding Social Research: Perspectives on Methodology and Practice. Falmer:London.

Bernard, H.R (1994): Research Methods in Anthropology: Qualitative and Quantitative Approaches. Second Edition, Chapter 10, 208-236. Sage Publications, Newbury Park, California.

Buxton M and Hanney S (1996) How Can Payback from Health Research be Assessed? Journal of Health Services Research and Policy, 1, 35-43.

Commonwealth Regional Health Community Secretariat (CRHCS) (2003) www.crhcs.or.tz 
ECDPM (2000): Using the Internet to share research and knowledge. InfoBrief 5, ECDPM Maastricht. http://www.ecdpm.org/pubs/infobrief5 gb.htm

Glaser, B. and Straus A. (1967) The Discovery of Grounded Theory. Chicago: Aldine

Haaga, J.G and Maru, R.M. (1996): The Effect of Operations Research on Program Changes in Bangladesh. Studies in Family Planning, vol. 27 (2), p76-87.

Hanney, S.R., Gonzales-Block, M.A., Buxton, M.J. and Kogan M. (2002) The Utilisation of Health Research in Policy-Making: Concepts, Examples and Methods of Assessment. A report to the Research Policy and Co-operation Department, World Health Organisation, Geneva. Health Economics Research Group Research (HERG) Report No. 28. HERG Brunel University, Uxbridge, UK.

Hanney, S, Packwood, T, Buxton, M (2000). Evaluating the Benefits from Health Research and Development Centres: A Categorization, A Model, and Examples of Application. Evaluation: The International Journal of Theory, Research and Practice, 6, 137-60.

Knott J and Wildavsky A. (1980) If dissemination is the answer, what is the problem? Knowledge, Creation, Diffusion, Utilisation, 1:537-78.

Kogan M and Tuijnman A. (1995) Educational Research and Development: Trends, Issues and Challenges. Paris, OECD.

Lavis, J. N (2003). Facilitating the Uptake of Research Knowledge by Health-System Managers and Public Policymakers: Some Lessons and Unresolved Challenges. WHO and Partners Meeting on Research Utilisation Guideline, WHO Offices, Geneva, 13-14 March 2003. 
Martin, A., Foreit,J.R., Ezcurra, E., and Vernon, R. (2002) Workshop on Utilisation of Research Results. San Jose, Costa Rica, June 4-7, 2002. WHO Dept Reproductive Health and Research, Population Council/FRONTIERS and Central America Population Centre University of Costa Rica.

Patton, M.Q., Grimes, P., Guthrie, K., Brennan, N., French K., and Blyth D. (1977). In search of impact: an analysis of the utilisation of federal health evaluation research. In Using Social Research in Public Policymaking (edited by C Weiss), 141-163, Lexington, Lexington Books.

Patton, M.Q (1990): Qualitative Evaluation and Research Methods. Second Edition. Sage Publications, Newbury Park, California.

Porter, R.W and Prysor-Jones, S. (1997): Making a Difference to Policies and Programmes: A Guide for Researchers. Support for Analysis and Research in Africa (SARA) Project. U.S Agency for International Development, Africa Bureau, Office of Sustainable Development. Washington, D.C.

RAND (1997): Population Matters: A RAND Program of Policy-relevant Research Communication. RAND, Santa Monica, California, USA.

Smith, R (1993): Filling the Lacuna between Research and Practice: An interview with Michael Peckham. British Medical Journal, vol. 307, 1403-7.

Solo J., Cerulli A., Miller R., Askew I., and Pearlman E. (1998). Strengthening the Utilisation of Family Planning Operations research: Findings from Case Studies in Africa. Population Council, AORTA Project II.

Taylor, S. J. and Bogdan, R. (1984). Introduction to Qualitative Research Methods: The search for meanings. New York: John Wiley \& Sons 
Torres, F.D (1981): How do Research Findings get used in the Development Plan? Paper presented at the $12^{\text {th }}$ Summer Seminar in Population, Makati, Manila, June 28-July 3, 1981.

Walt, G (1994): Health Policy: An introduction to Process and Power. Zed Books, London.

Walt, G (1994b): How far does Research Influence Policy? European Journal of Public Health, vol. 4, p233-235.

Weiss, C. (1991) Policy Research: data, ideas or arguments? In Social Sciences and Modern States (Eds. Wagner, P. Weiss, C Wittrock B, Wolman H) Cambridge University Press,:307332.

Weiss, C (1979): The Many Meanings of Research Utilisation. Public Administration Review, vol. $39,426-31$.

Weiss, C (1977) Introduction (1-22) in Using Social Research in Public Policymaking (edited by C Weiss) Lexington, Lexington Books.

Worral, R (1972): Problems of Collection and Dissemination of Population Information and Knowledge in Asia. Paper presented at the Economic Commission for Asia and the Far East, Second Asian Population Conference, Tokyo, November 1-13, 1972.

White, K (1993): We Couldn't Have Asked for More: Lessons Learned in Information Dissemination. Technologies for Primary Health Care (PRITECH), Occasional Operations Papers. Management Sciences for Health, Arlington, Virginia.

WHO (2000): Promoting Evidence-based Reproductive Health. Progress In Reproductive Health Research, No. 54, Part 1. 


\section{Acknowledgements}

This work was completed under a Small Grant from the Opportunities and Choices Reproductive Health Research Programme, University of Southampton, UK, funded by the Department for International Development (DfID). The authors would like to thank all those who contributed to this research in the study countries. 